\title{
As Técnicas de Reflectância Difusa de Fotólise por Pulso de Laser e de Estado Fundamental ou Como Estudar Reacções Fotoquímicas em Superfícies de Sólidos
}

\author{
LUÍS FILIPE VIEIRA FERREIRA *a, JOSÉ CARLOS NETTO-FERREIRAb,
} ANABELA S. OLIVEIRAa, SÍlVIA M. B. COSTAC

\section{INTRODUÇÃO}

A Fotólise de Relâmpago (vulgarmente designada por "flash-fotólise") foi desenvolvida nos anos 50 por G. Porter [1] (Prémio Nobel da Química em 1967) e revelou-se uma técnica única para o estudo de reacções em amostras transparentes e homogéneas, nomeadamente em solução diluída, fase gasosa e em meio rígido. Tornou-se assim possível (ver Figura 1) a detecção de espécies transientes, isto é, moléculas ou fragmentos destas com tempos de vida curtos, gerados após uma excitação inicial por um pulso muito curto de luz ultravioleta ou visível (uma vez que a maior parte dos compostos absorve radiação nestes comprimentos de onda), originando a formação de espécies electronicamente excitadas. Este excesso de energia pode dar origem a reacções químicas. de detectores de matrizes de díodos permitem o traçado de espectros de absorção resolvidos no tempo das espécies excitadas. A fotólise de relâmpago convencional limita-se à escala temporal do microsegundo imposta pela duração do relâmpago de excitação inicial.

A utilização de laseres pulsados, equipados com sistemas electroópticos de disparo (Q-switch lasers) permitiu uma excitação mil vezes mais rápida que a convencional (a duração de um pulso laser é de cerca de 8 ns num laser de Neodímio-YAG) e proporcionou uma grande evolução no estudo de cinéticas de decaimento que ocorrem na gama temporal do nanosegundo. A deteç̧ão pode ser feita com fotomultiplicador ou fotodetectores rápidos acoplados a osciloscópios rápidos que digitalizam os transientes nesta gama temporal.

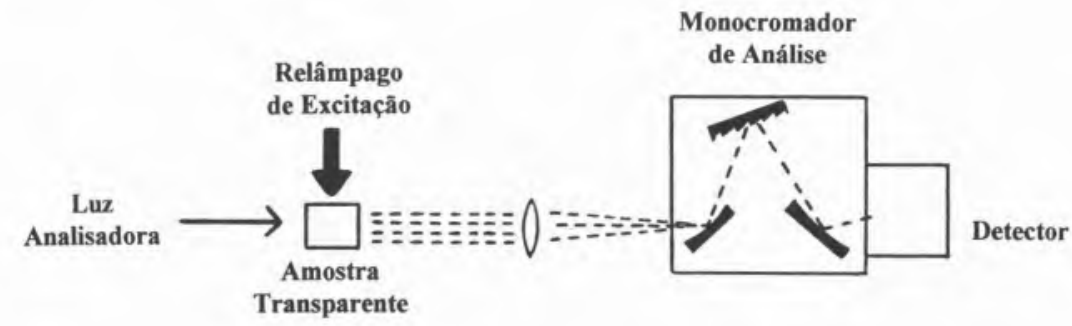

Figura 1 - Diagrama de um aparelho de fotólise de relâmpago "convencional" com geometria de transmissão.

Na fase inicial de desenvolvimento da técnica esse pulso era o disparo de lâmpadas de relâmpago com duração de fracções de segundo. As espécies transientes assim produzidas são observadas (com o auxílio de uma luz analisadora gerada com um atraso conhecido em relação ao relâmpago inicial), por absorção óptica (cinética ou espectrográfica). Hoje em dia usam-se correntemente pulsos de laser com duração de nanosegundo ou até fraç̧ões deste para a geração inicial das espécies excitadas. A utilização de monocromadores de análise e fotomultiplicadores apropriados (normalmente sensíveis na gama de 200 a $900 \mathrm{~nm}$ ) e mais recentemente o uso
É na gama temporal do nanosegundo que se estudam muitas reacções fotoquímicas que envolvem processos bimoleculares puramente controlados por difusão ou com componentes estáticas. A transferência de energia de ressonância, a permuta electrónica ou a transferência electrónica podem em muitos casos ser quantificadas com o auxílio desta técnica.

Na fotólise de pulso de laser de picosegundo $\left(10^{-12} \mathrm{~s}\right)$ a radiação de excitação é fornecida por um laser "mode-locked". As unidades de atraso baseiam-se na variação do percurso óptico uma vez que a luz se desloca apenas $0.3 \mathrm{~mm}$ em cada ps. Assim se conseguem pulsos de excitação e análise ("pump and probe") separados de apenas alguns picosegundos, os quais depois se detectam e registam com sistemas de matrizes de díodos com portas na gama temporal apropriada. A variação do atraso temporal permite a obtenção de espectros resolvidos no tempo.

Nesta escala temporal estudamse muitos fenómenos importantes em fotoquímica, como por exemplo a reorganização do solvente em torno de uma molécula excitada, a relaxação vibracional, mecanismos de transferência de electrão ou de protão e reaç̧ões unimoleculares, entre outros.

A fotoquímica de fentosegundo $\left(10^{-15} s\right)$ estuda usualmente reaç̧ões de dissociação e de isomerização em fase gasosa e ainda a transferência de protão intramolecular e isomerizações fotocrómicas que ocorrem nesta gama temporal em solução.

\section{A FOTÓLISE POR PULSO DE LASER EM MODO DE REFLECTÂNCIA DIFUSA}

Existe actualmente um interesse considerável no estudo de propriedades fotoquímicas de sistemas heterogéneos, muitos dos quais são opacos. Exemplos disso são muitos estudos em fotobiologia, as reacções fotoquímicas em espaços confinados, estudos de catálise, estudos de corantes adsorvidos ou covalentemente ligados a fibras naturais e sintéticas, entre muitos outros.

Tudo indica que a reflectância difusa de laser venha a ter, no estudo de reacções fotoquímicas em meio heterogéneo, um papel pelo menos tão importante como o que a fotólise em modo de transmissão tem tido para o estudo de reacções fotoquímicas em meio homogéneo.

No início dos anos 80, F. Wilkinson e colaboradores conseguiram demonstrar que a técnica de fotólise de pulso de laser podia também ser aplicada a amostras opacas [2], estudando as modificações introduzidas pela absorção dos transientes gerados 


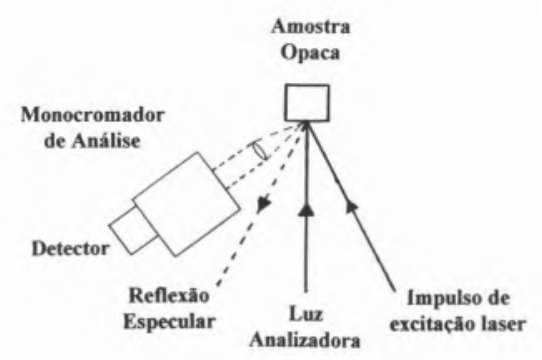

Figura 2 - Diagrama esquemático mostrando a geometria usada na fotólise de pulso de laser em reflectância difusa.

pelo pulso de um laser na luz analisadora detectada em modo de reflectância difusa (ver Figura 2).

O equipamento usado é idêntico ao dos estudos em transmissão, mas agora utiliza-se uma geometria de reflexão, encontrando-se a luz analisadora e a luz detectada do mesmo lado da amostra. Tal como na fotólise de relâmpago convencional os transientes agora obtidos são espectros de diferença, isto é, a absorção determinada experimentalmente reflecte a diferença de absorção no estado excitado e no estado fundamental para uma dada espécie a cada comprimento de onda analisado. Assim, a \% de absorção determinada pode ser positiva ou negativa consoante o estado excitado ou o estado fundamental tenham o coeficiente de extinção molar superior, respectivamente. Daqui resulta o aparecimento de pontos isosbésticos bem definidos em sistemas simples.

De facto, considerando que I e $\mathrm{J}$ são os fluxos de luz incidente e dispersa, o fluxo incidente, I, diminui à medida que penetra na amostra sólida quer porque há absorção de radiação quer porque as partículas dispersam a luz; por outro lado, I é aumentado com a dispersão de J. O fluxo de luz dispersa, J, emergente tem uma variação análoga mas no sentido oposto. Considerando os coeficientes de absorção, K, e de dispersão, S, Kubelka e Munk estabeleceram em 1948 que para um difusor ideal e para amostras opticamente densas, ou seja, todas aquelas em que um aumento da es- pessura não faz variar a reflectância da amostra, a reflectância $\mathrm{R}$ é dada por:

$$
\mathrm{R}=\mathrm{J} / \mathrm{I}_{\mathrm{o}}
$$

e $\mathrm{R}$ está relacionada com $\mathrm{K}$ e $\mathrm{S}$ pela função de remissão, $F(R)$

em que

$$
F(R)=(1-R)^{2} /(2 R)=K / S
$$

$$
K(\lambda)=2 \varepsilon(\lambda) C .
$$

onde $\varepsilon$ é o coeficiente de absorção neperiano e $\mathrm{C}$ a concentração da espécie absorvedora.

A função de remissão varia linearmente com o número de cromóforos que absorvem na amostra sólida considerados uniformemente distribuídos. $\mathrm{K}$ e $\mathrm{S}$ são independentes da profundidade de penetração da luz na amostra.

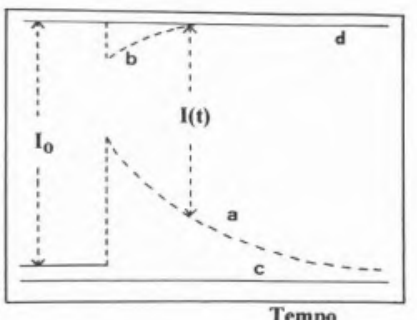

Figura 3a) Lei de Beer para soluções - $\log I(t) / I(o) \alpha$ Concentração de transiente. a - transiente de absorção (disparo do laser $\mathrm{e}$ lâmpada).

b - transiente de emissão (só disparo do laser). c - linha de base.

d - linha de topo (zero do sistema)

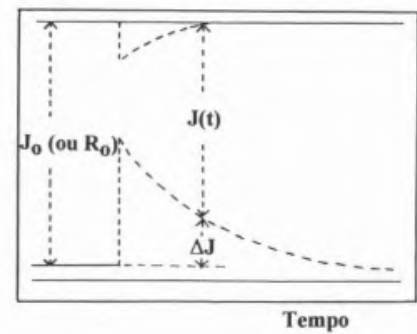

Figura 3b) Amostras sólidas difusoras de luz [Jo- I (t)I/lo $\alpha$ Conc, de transiente ou $\Delta R(t)=$ $[R o-R(t)] / R o=(1-R) \alpha$ Conc. de transiente.
Para pequenas absorções, $\mathrm{R} \sim 1 \mathrm{e}$ então

$$
(1-R)^{2} /(2 R) \sim(1-R)
$$

Nestas condições uma representação de (1-R) em função do tempo é uma medida da concentração do transiente para amostras pouco concentradas (para amostras mais concentradas deve usar-se $\Delta \mathrm{F}(\mathrm{R}))$.

A Figura 3 compara os sinais obtidos na fotólise com geometria convencional de transmissão e na fotólise de pulso de laser em reflectância difusa.

Para o caso de amostras mais concentradas a variação da função de remissão antes e depois do disparo do laser é

$$
\Delta \mathrm{F}(\mathrm{R}(\mathrm{t}))=\mathrm{F}(\mathrm{R}(\mathrm{t}))-\mathrm{F}(\mathrm{R}(0))(5)
$$

e como $\mathrm{K}(\mathrm{t})=\mathrm{F}(\mathrm{R}(\mathrm{t})) . \mathrm{S}=\mathrm{K}_{\mathrm{B}}+$ $2 \varepsilon_{\mathrm{G}} \mathrm{C}_{\mathrm{G}}+2 \varepsilon^{*} \mathrm{C}^{*}, \mathrm{~K}(0)=\mathrm{F}(\mathrm{R}(0)) \cdot \mathrm{S}=$ $\mathrm{K}_{\mathrm{B}}+2 \varepsilon_{\mathrm{G}} \mathrm{C}^{\circ}$ e $\mathrm{C}^{\circ}=\mathrm{C}_{\mathrm{G}}+\mathrm{C}^{*}$, em que $\mathrm{G}$ representa o estado fundamental, $\mathrm{K}_{\mathrm{B}} \mathrm{O}$ coeficiente de absorção do substrato e o asterisco designa o estado excitado, vem imediatamente

$$
\Delta \mathrm{F}(\mathrm{R}(\mathrm{t}))=2\left(\varepsilon^{*}-\varepsilon_{\mathrm{G}}\right) \mathrm{C}^{*} / \mathrm{S}
$$

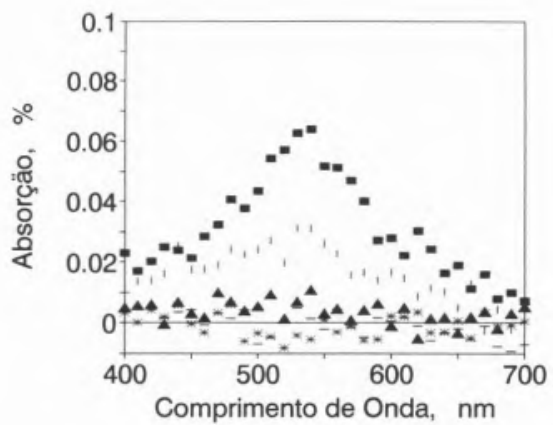

Figura 4 - Espectro de absorção resolvido no tempo de uma amostra $0.75 \mathrm{mmol} \mathrm{g}^{-1}$ de benzofenona depositada na superfície de celulose microcristalina após o disparo do laser a $355 \mathrm{~nm}$. $(\%$ Absorção= $(1-\mathrm{J}(\mathrm{t}) / \mathrm{Jo}) \times 100)$. O solvente usado na preparação da amostra foi o diclorometano $\left(\mathrm{CH}_{2} \mathrm{Cl}_{2}\right)$. A curva ( $\left.\mathbf{m}\right)$ foi obtida 1 microsegundo após o disparo do laser, (|) 2.5 microsegundos, (A) 10 microsegundos, (-) 25 microsegundos, (.) 75 microsegundos. 
o que justifica o aparecimento dos pontos isosbésticos referidos anteriormente.

Os decaimentos apresentados na Figura 3b) dizem respeito a um dado comprimento de onda de análise. Considerando todos os comprimentos de onda onde a espécie excitada absorve e escolhendo tempos de análise apropriados, na curva de decaimento podem construir-se espectros como o da Figura 4 que nos dão em simultâneo informação espectroscópica e cinética das amostras opacas.

\section{REACCCÕES INDUZIDAS PELA LUZ EM SISTEMAS HETEROGÉNEOS}

O espectro apresentado na Figura 4 é o da benzofenona depositada à superfície de celulose microcristalina usando um solvente apolar, o benzeno, e a concentração nominal é de 0.75 mmole por grama de substrato.

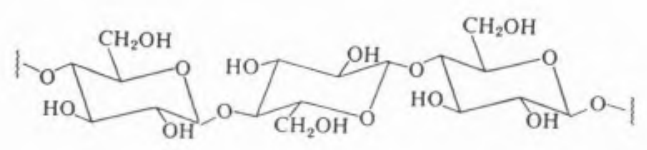

Figura 5 - Estrutura da celulose microcristalina.

Este espectro é em tudo idêntico ao obtido com cristais de benzofenona, com um máximo a $525 \mathrm{~nm}$; apenas a percentagem de absorção é menor dado que a quantidade de cetona a absorver é também menor. É pois lógico admitir que a benzofenona está sob a forma de microcristais e não em contacto íntimo com as cadeias do polímero natural, a celulose.

A Figura 5 mostra a fórmula estrutural da celulose microcristalina.

Estruturalmente a celulose é um polímero da D-glucose no qual as unidades individuais estão unidas por ligações $\beta$-glicosídicas entre o carbono anomérico de uma das uni- dades e o grupo hidroxilo em $\mathrm{C}_{4}$ da unidade seguinte. A celulose é provavelmente o composto orgânico mais abundante na terra. É o principal componente estrutural das células vegetais. A resistência da madeira provém principalmente das ligações de hidrogénio entre os grupos hidroxilo de uma cadeia e os das cadeias vizinhas. Estas ligações de hidrogénio são muito favorecidas pela estrutura linear do polímero natural com conformaçōes que podem ser rectilíneas (ao contrário do que se verifica no amido onde também por motivos estruturais as cadeias embora possam ser lineares não podem adquirir uma conformação rectilínea).

Estudos de raios - $\mathrm{X}$ mostraram que a celulose nativa é um sistema de duas fases: uma amorfa, menos ordenada e compacta, localizada à superfície das fibrilas elementares; outra ordenada e compacta (cristalitos) onde as cadeias existem numa forma cristalina definida e fortemente ligadas por ligações de hidrogénio às cadeias mais próximas.

A celulose microcristalina não é mais do que uma forma pura da celulose obtida por um tratamento ácido da celulose nativa. As re-

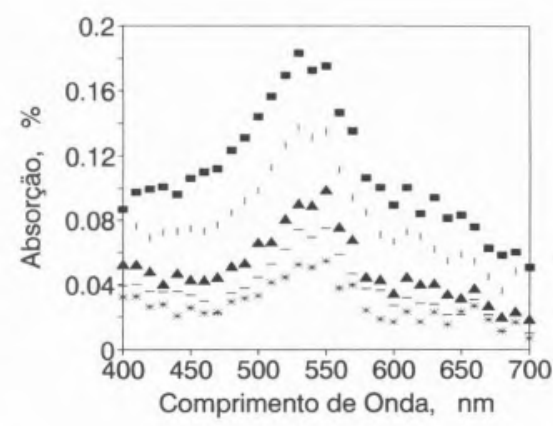

Figura 6 - Espectro de absorção resolvido no tempo de uma amostra $0.75 \mathrm{mmol} \mathrm{g}^{-1}$ de benzofenona adsorvida em celulose microcristalina. $O$ solvente usado na preparação da amostra foi o metanol. A curva ( $\mathbf{m}$ ) foi obtida 1 microsegundo após o disparo do laser, (|) 2.5 microsegundos, (A) 10 microsegundos, (-) 25 microsegundos, (.) 75 microsegundos. giões amorfas são preferencialmente atacadas e transformadas, sendo o resíduo final altamente cristalino.

É bem conhecida a propriedade que as fibras de celulose possuem de incharem (e esticarem) na presença de humidade. Todos nós sabemos que uma camisola de algodão estica nos dias mais húmidos. Outros solventes polares próticos ou apróticos como o metanol, o etanol, o acetonitrilo e a acetona também promovem o inchamento da celulose microcristalina. No entanto solventes como o benzeno, o tolueno ou o diclorometano não conseguem afastar as cadeias do polímero natural. É assim possível controlar a adsorção de moléculas na celulose microcristalina, à superfície no segundo caso e no seio das cadeias no caso dos solventes que incham o polímero. Após remoção do solvente usado na preparação das amostras é promovida uma interacção cadeia-hóspede-cadeia que substitui a interacção cadeia-solvente-cadeia anteriormente existente.

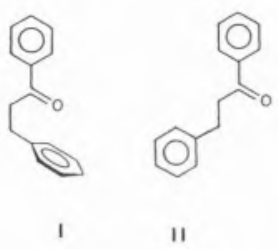

Figura 7 - Conformações da $\beta$-Fenilpropiofenona.

A Figura 6 mostra a ocorrência da transformação da benzofenona excitada em radical cetilo por abstracção de um átomo de hidrogénio da cadeia polimérica ( $\mathrm{H}$ ligados a carbonos secundários que também possuem um grupo hidroxilo) [3a].

O radical cetilo tem um tempo de vida bem mais longo do que o tripleto da benzofenona, ocorrendo o máximo de absorção a $545 \mathrm{~nm}$. Outras reacções de abstracção de hidrogénio por cetonas excitadas foram já 
estudadas usando esta técnica [3b], nomeadamente a $\beta$-fenilpropiofenona e derivados da acetofenona, moléculas modelo no estudo do problema do amarelecimento do papel por acção da luz solar.

Um exemplo interessante de restrições conformacionais impostas pela geometria do hospedeiro é dado pela $\beta$-fenilpropiofenona ( $\beta$ PP) quando incluída no zeólito hidrofóbico silicalite [3c]. A molécula pode existir nas conformações I e II.

Em solução a molécula é muito estável do ponto de vista fotoquímico mesmo em solventes que sejam bons doadores de hidrogénio, no que contrasta com outras cetonas análogas como a benzofenona (BZP) e a acetofenona (ACP) que no estado excitado formam prontamente o radical cetilo. Esse comportamento da $\beta$-PP está ligado ao facto de esta poder facilmente assumir em meio homogéneo a conformação I em que ocorre uma extinção intramolecular do carbonilo excitado. O tempo de vida do tripleto é de cerca de lns, insuficiente para se dar a reacção de abstracção do átomo de hidrogénio de um modo eficiente.

Quando incluída nos canais da silicalite o tripleto da $\beta$-PP vive alguns microsegundos, pois a conformação I é impedida. O mesmo acontece quando a $\beta$-PP é adsorvida em celulose microcristalina após inchamento com um solvente como o etanol ou o metanol. Neste caso e em contraste com o comportamento em solução forma-se o cetilo da $\beta$-PP [3b].

\section{OS ESPECTROS DE REFLECTÂNCIA DIFUSA (UV-VIS-IVP) NO ESTADO FUNDAMENTAL}

Os espectros de absorção do estado fundamental de amostras sólidas opacas podem fazer-se como se fazem os espectros de absorção de amostras transparentes. No segundo caso usa-se a lei de Beer e determi-

\title{
DATALAB SOLUTION DA LABTECH
}

\author{
Software em Ambiente Windows (NoteBook)
}

e Placa de Aquisição e Controlo ( 8 canais Analog.In, 2 Analog.Out + 8 Digit.In +8 Digit.Out)

por apenas $110000 \$ 00+\mathrm{IVA}$
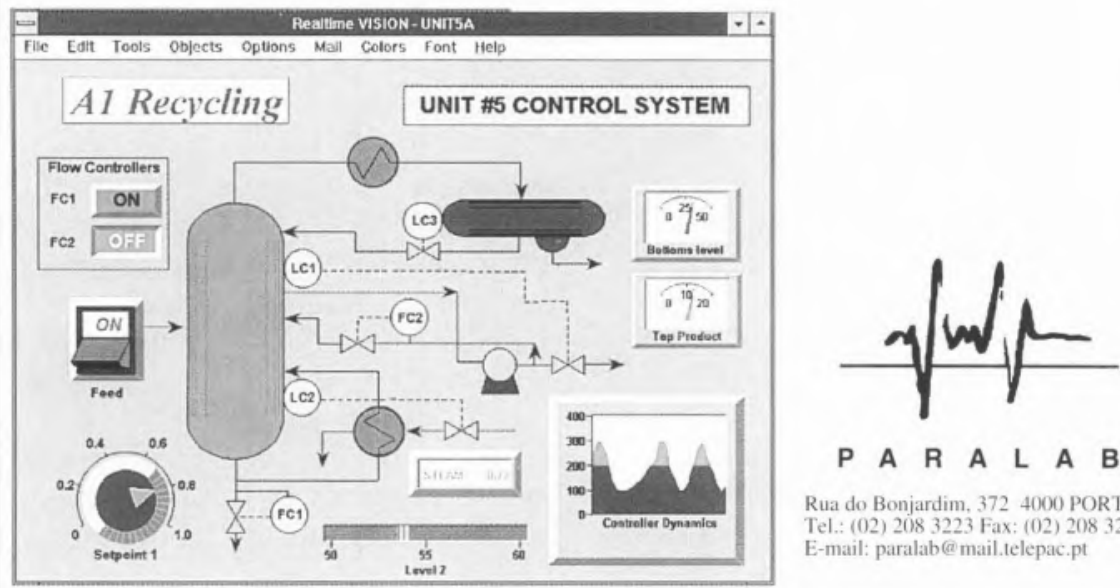

P A R A L A B

Rua do Bonjardim, 3724000 PORTO E-mail: paralab@mail.telepac.pt

nam-se as absorvâncias em função do comprimento de onda. No caso de amostras sólidas determina-se a Reflectância R em função do comprimento de onda, após calibração do sistema. Um difusor ideal tem $\mathrm{R}=1.0$ (por exemplo o sulfato de bário ou o óxido de magnésio muito puros apresentam $\mathrm{R} \sim 0.980 \pm 02$ na gama de 200 a $900 \mathrm{~nm}$ ) e o carvão em partículas finas apresenta $\mathrm{R} \sim 0$. Outra alternativa é o recurso a padrões calibrados para o branco e para o preto que se vendem comercialmente. A celulose microcristalina apresenta reflectâncias que se aproximam da unidade no visivel (VIS) e infravermelho próximos (IVP) mas têm absorções significativas (pequenas reflectâncias) no ultra-violeta (UV). Se uma amostra tiver mais de um cromóforo a absorver, para amostras opticamente densas podemos escrever

$K(\lambda)=K_{\text {substrato }}+2 \sum_{i} \varepsilon_{i}(\lambda) C_{i}$ pelo que uma reacção fotoquímica após irradiação do cromóforo adsorvido pode seguir-se temporalmente por um espectro de diferença

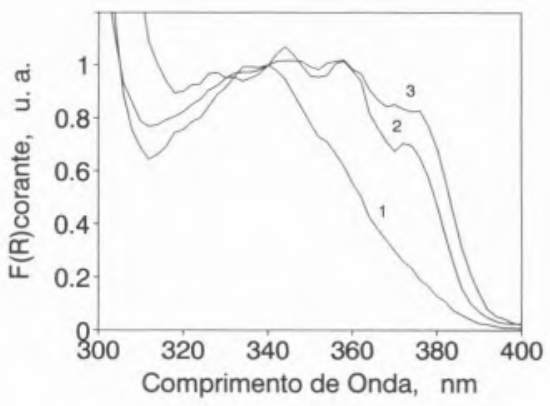

Figura 8 - Espectro de absorção no estado fundamental da benzofenona adsorvida em celulose microcristalina $(0.75 \mathrm{mmol} \mathrm{g}-1)$. As ordenadas são a função de remissão em unidades arbitrárias. Os solventes usados na preparação das amostras foram: curva (1)- metanol; curva (2) - diclorometano. A curva (3) refere-se a uma mistura mecânica com a mesma quantidade de benzofenona. 
$\Delta K=S\left[F(R)_{\text {irradiado }}-F(R)_{\text {não irradiado }}\right]$

Outra aplicação interessante desta técnica mostra-se na Figura 8 onde se apresentam os espectros das amostras de benzofenona usadas para a obtenção dos espectros das figuras 4 e 6 , focando as nossas atenções nas transições $S_{0} \rightarrow S_{1}$ da benzofenona com carácter $n, \pi^{*}$ pois a absorção de um fotão a $355 \mathrm{~nm}$ origina neste caso a promoção de um electrão não ligante do oxigénio do carbonilo a uma orbital $\pi$ antiligante.

É bem conhecido em fotoquímica, dos estudos em solução, que solventes como o etanol e outros solventes polares próticos promovem um deslocamento para o azul da transição $n, \pi^{*}$ do carbonilo da BZP pois o oxigénio do carbonilo é mais fortemente ligado por ligações de hidrogénio no estado fundamental do que no estado excitado. $\mathrm{O}$ que este espectro mostra é que em amostras preparadas em etanol a BZP está inserida no seio da celulose microcristalina com forte interacção com os grupos hidroxilo das cadeias celulósicas. Pelo contrário a comparação das amostras

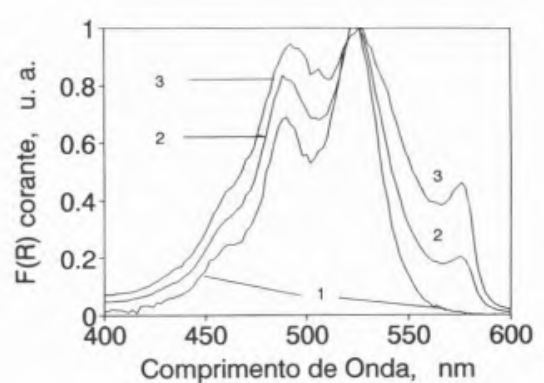

Figura 9 - Espectro de absorção no estado fundamental da 1,1'-Dietil-2,2'-Cianina adsorvida em celulose microcristalina. As curvas estão normadas no máximo de absorção do monómero. As concentrações são: (1) 0.01 micromoles por grama; (2) 5 micromoles por grama; (3) 15 micromoles por grama.

Palavras chave: Reflectância Difusa, Fotólise de Relâmpago Convencional, Fotólise por Pulso de Laser, Cetonas, Silicalite, Cianinas. preparadas com diclorometano com as obtidas por mistura mecânica é reveladora da formação de cristalitos depositados à superfície da celulose que não sofreu inchamento.

Outro exemplo importante da aplicação desta técnica é o dado pelo estudo da formação de agregados de corantes no seio de polímeros naturais ou sintéticos.

As cianinas são em muitos casos corantes catiónicos, com larga aplicação nas indústrias têxtil e fotográfica. Mais recentemente iniciou-se o seu uso como sensibilizador na fototerapia do cancro. A $1,1^{\prime}$ - dietil - 2,2' - cianina adsorvida em celulose microcristalina em baixas concentrações $(\approx 0.001$ a 0.1 micromoles por grama de substrato), apresenta um espectro de reflectância difusa que é muito semelhante ao espectro de absorção do mesmo composto em solução etanólica. No entanto em concentrações superiores (gama de $\approx 1$ a 20 micromoles de corante por grama de substrato) este corante evidencia a formação de dímeros em sanduíche (agregados H) e ainda agregados cabeça-cauda (agregados $\mathrm{J}$ ) como se mostra na Figura 9. A formação destes últimos leva ao aparecimento de uma nova banda de absorção que é estreita e ocorre a maiores comprimentos de onda, reforçando assim a absorção do corante na gama espectral das menores energias. O dímero em sanduíche absorve a maiores energias que o correspondente monómero do corante.

\section{5 - ALGUMAS NOTAS FINAIS}

Apresentaram-se alguns exemplos de aplicação da técnica de fotólise por pulso de laser em modo de reflectância difusa a sistemas heterogéneos. Os espectros de absorção e emissão resolvidos no tempo obtidos por esta técnica proporcionam uma análise espectroscópica e cinética que permite um estudo seguro de muitas reacções fotoquímicas que ocorrem à superfície dos sólidos. A conjugação desta informação com a obtida no estudo da absorção no estado fundamental (antes e depois da irradiação das amostras sólidas em muitos casos) permite frequentemente obter informações que não estariam acessíveis por nenhuma das técnicas isoladamente.

Os processos fotoquímicos primários podem agora ser estudados em sistemas opacos e heterogéneos com o uso destas técnicas, sem ser necessário recorrer ao uso dos sistemas modelo clássicos ou seja as amostras transparentes em solução.

* A quem a correspondência deve ser dirigida.

a) Centro de Química Física Molecular, Complexo I, IST, Av. Rovisco Pais, 1096 Lisboa Codex, Portugal.

b) Departamento de Química, Universidade Federal Rural do Rio de Janeiro, Rio de Janeiro, CEP 23460, Brasil.

c) Centro de Química Estrutural, Complexo I, IST, Av. Rovisco Pais, 1096 Lisboa Codex, Portugal.

REFERÊNCIAS

1. G. Porter - Proc. R. Soc., A200 (1950) 284.

2. F. Wilkinson, G.P. Kelly - "Handbook of Organic Photochemistry", Ed. I.C. Scaiano, CRC Press, Boca Raton, Vol.1. (1990) p.293.

3. a) L.F. Vieira Ferreira, I.C. Netto-Ferreira, I.V. Khmelinskii, A.R. Garcia, S.M.B. Costa - Langmuir, 11 (1995) 231 b) L.F. Vieira Ferreira, I.C. Netto-Ferreira, S.M.B, Costa Spectrochimica Acta, 51A (1995) 231, c) L.F. Vieira Ferreira, I.C. Netto-Ferreira, S.M.B. Costa - Quim. Nova, (1996) In Press; d) L.F Vieira Ferreira, A.S. Oliveira, F. Wilkinson, D.R. Worrall - I. Chem. Soc. Faraday Trans., (1996) In Press. 